\title{
INFLUÊNCIA DA TEMPERATURA E DA CONCENTRAÇÃO NA MASSA ESPECÍFICA DE XAROPES DESTINADOS À PRODUÇÃO DE AÇÚCAR E BIOETANOL
}

\author{
César Augusto CANCIAM*
}

\begin{abstract}
*Mestre em Engenharia Química, professor lotado no Departamento Acadêmico de Engenharia Química da Universidade Tecnológica Federal do Paraná - Câmpus Ponta Grossa, canciam@utfpr.edu.br
\end{abstract}

Recebido em: 10/08/2015 - Aprovado em: 06/01/2016 - Disponibilizado em: 30/07/2016

\begin{abstract}
RESUMO:
A massa específica é uma propriedade termofísica usada na caracterização dos materiais. O objetivo desse trabalho foi avaliar a influência da temperatura e da concentração sobre a massa específica de xaropes destinados à produção de açúcar e bioetanol, entre 20 e $80^{\circ} \mathrm{C}$. Foram consideradas as concentrações de 60,65 e $70^{\circ}$ Brix, nas purezas de 80 , 85 e $90 \%$. Para cada concentração, foi encontrada uma relação do tipo Arrhenius. Os valores encontrados para a energia de ativação variaram de $380,7139\left(70^{\circ}\right.$ Brix com $80 \%$ de pureza) a $390,4421 \mathrm{~J} \mathrm{~mol}^{-1}\left(60^{\circ} \mathrm{Brix}\right.$ com $90 \%$ de pureza). Os resultados sugerem que com o aumento da concentração, para uma mesma pureza, a energia de ativação para a massa específica diminui. E à medida em que a temperatura aumenta, diminui a massa específica. Na literatura, não foram encontrados valores experimentais da energia de ativação para a massa específica desse tipo de material, o que dificultou a comparação dos resultados.

PALAVRAS-CHAVE: Massa específica. Xarope. Efeito. Temperatura. Concentração.
\end{abstract}

\section{Influence of temperature and concentration on the specific weight of syrups for the production of sugar and bioethanol}

\begin{abstract}
:
The specific weight is a thermophysical property used for characterization of materials. The objective of this work was evaluate the influence of temperature and concentration on the specific weight of syrups for the production of sugar and bioethanol, between 20 and $80^{\circ} \mathrm{C}$. Concentrations were 60, 65 and $70^{\circ}$ Brix, with the purities of 80,85 and $90 \%$. For each concentration, it was found an Arrhenius-type relationship. The values found for the activation energy ranged from $380.7139\left(70^{\circ}\right.$ Brix with $80 \%$ purity) to $390.4421 \mathrm{~J} \mathrm{~mol}^{-1}\left(60^{\circ}\right.$ Brix with $90 \%$ purity). The results suggest that with increasing concentration, for the same purity, the activation energy for the density decreases. And as the temperature increases, the specific weight decreases. In the literature, there are no experimental values of the activation energy for specific weight for this material, making it difficult to compare results.

KEYWORDS: Specific weight. Syrup. Effect. Temperature. Concentration.
\end{abstract}

\section{INTRODUÇÃO}

São consideradas como propriedades termofísicas dos materiais as grandezas: massa específica, viscosidade, capacidade calorífica, condutividade térmica e difusividade térmica. $\mathrm{O}$ conhecimento dessas grandezas é importante em muitas aplicações nas áreas da Ciência e da Engenharia. Em particular, nos cálculos relacionados à Termodinâmica, à Mecânica dos Fluidos e nas Transferências de Calor e de Massa subjacentes às Operações Unitárias (MATOS, 1998; MAGERROMOV et al., 2008). 
A massa específica, no caso de líquidos, reflete o grau de empacotamento de suas espécies químicas, que é decorrência da intensidade das interações entre essas espécies. Dessa forma, interações mais intensas tende a se relacionar com massas específicas maiores. Como volume e massa específica são grandezas inversamente proporcionais. Quanto mais fortes as interações, mais empacotadas estão as espécies químicas, portanto o volume será menor e consequentemente a massa específica será maior (BROWN e HOLME, 2009).

Os mesmos autores comentam que quanto maior a temperatura de um líquido, maior o movimento cinético de suas espécies químicas e mais intensa a agitação entre elas. Isto provoca um cisão das interações estabelecidas entre as espécies químicas e o afastamento delas, com consequente aumento de volume.

Equações do tipo Arrhenius permitem verificar o efeito da temperatura sobre um determinado parâmetro físico-químico. Dos parâmetros físico-químicos, Equações do tipo Arrhenius são escritas para a viscosidade absoluta, massa específica e coeficiente de difusividade efetiva (TSEN e KING, 2002; GIAP, 2010; CHEN, ZHENG e ZHU, 2012).

A Equação 1 corresponde a uma Equação do tipo Arrhenius e considera a massa específica $(\rho)$ em função da temperatura absoluta ( $T$ ) (TSEN e KING, 2002; GIAP, 2010; CANCIAM, 2014):

$$
\rho=\rho_{\infty} \cdot \exp \left(\frac{E_{a}}{R \cdot T}\right)
$$

Os termos $\rho_{\infty}$ e $E_{a}$ correspondem, respectivamente, à massa específica quando a temperatura tende ao infinito e à energia de ativação para a massa específica. $\mathrm{O}$ termo $R$ equivale à constante universal dos gases ideais.

A energia de ativação para a massa específica $\left(E_{a}\right)$ indica a sensibilidade da massa específica devido à variação da temperatura. Ou seja, valores elevados dessa grandeza indicam uma mudança mais rápida na massa específica com a temperatura (CANCIAM, 2014).

A cana-de-açúcar é a principal matéria-prima para a indústria sucroalcooleira brasileira. A produção de açúcar e bioetanol envolve várias etapas, dentre elas destaca-se a evaporação do caldo (MEZAROBA, MENEGUETTI, GROFF; 2010; JÚNIOR et al., 2013).

Mezaroba, Meneguetti e Groff (2010) comentam que a evaporação do caldo é realizada para que a concentração do xarope fique entre 60 e $70^{\circ}$ Brix, sendo mais recomendado o valor de $65^{\circ}$ Brix. $\mathrm{Na}$ evaporação do caldo, cerca de $80 \%$ em peso do caldo é removida na forma de água evaporada.

O objetivo desse trabalho foi avaliar a influência da temperatura e da concentração 
na massa específica de xaropes produzidos a partir da evaporação do caldo.

\section{MATERIAIS E MÉTODOS}

Para a determinação da massa específica dos xaropes utilizou-se o software de domínio público disponibilizado na página da internet da associação "The Sugar Engineers". Esse software foi desenvolvido com base no trabalho de Bubnik et al. (1995) e teve como dados de entrada: temperatura, concentração e pureza.

A faixa de temperatura considerada foi de 293,15 a $353,15 \mathrm{~K}$ (de 20 a $80^{\circ} \mathrm{C}$ ), com uma variação de $5 \mathrm{~K}$ (ou $5^{\circ} \mathrm{C}$ ) entre uma temperatura e outra. Foram consideradas as concentrações de 60,65 e $70^{\circ}$ Brix, nas purezas de 80,85 e $90 \%$.

A Tabela 1 relaciona os valores estimados da massa específica do xarope com $60^{\circ}$ Brix, nas purezas de 80,85 e $90 \%$.

Tabela 1. Massa específica estimada $\left(\mathrm{em} \mathrm{kg} \cdot \mathrm{m}^{3}\right)$ do xarope com $60^{\circ}$ Brix em função da pureza

\begin{tabular}{cccc}
\hline $\begin{array}{c}\text { Temperatura } \\
(\mathrm{K})\end{array}$ & $\begin{array}{c}\text { Pureza } \\
80 \%\end{array}$ & $\begin{array}{c}\text { Pureza } \\
85 \%\end{array}$ & $\begin{array}{c}\text { Pureza } \\
90 \%\end{array}$ \\
\hline 293,15 & 1287,5 & 1317,6 & 1348,8 \\
298,15 & 1285,0 & 1315,1 & 1346,2 \\
303,15 & 1282,5 & 1312,5 & 1343,5 \\
308,15 & 1279,8 & 1309,8 & 1340,7 \\
313,15 & 1277,1 & 1307,0 & 1337,9 \\
318,15 & 1274,4 & 1304,2 & 1335,1 \\
323,15 & 1271,5 & 1301,3 & 1332,2 \\
328,15 & 1268,6 & 1298,3 & 1329,2 \\
333,15 & 1265,6 & 1295,3 & 1326,1 \\
338,15 & 1262,5 & 1292,2 & 1323,0 \\
343,15 & 1259,4 & 1289,1 & 1319,9 \\
348,15 & 1256,1 & 1285,9 & 1316,7 \\
353,15 & 1252,9 & 1282,6 & 1313,4 \\
\hline
\end{tabular}

Fonte: Autor.
As Tabelas 2 e 3 relacionam os valores estimados da massa específica dos xaropes com 65 e $70^{\circ}$ Brix, respectivamente.

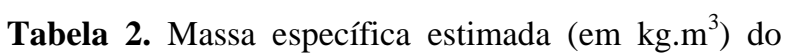
xarope com $65^{\circ}$ Brix em função da pureza

\begin{tabular}{cccc}
\hline $\begin{array}{c}\text { Temperatura } \\
(\mathrm{K})\end{array}$ & $\begin{array}{c}\text { Pureza } \\
80 \%\end{array}$ & $\begin{array}{c}\text { Pureza } \\
85 \%\end{array}$ & $\begin{array}{c}\text { Pureza } \\
90 \%\end{array}$ \\
\hline 293,15 & 1287,2 & 1317,3 & 1348,4 \\
298,15 & 1284,7 & 1314,7 & 1345,7 \\
303,15 & 1282,2 & 1312,1 & 1343,0 \\
308,15 & 1279,6 & 1309,4 & 1340,3 \\
313,15 & 1276,9 & 1306,7 & 1337,5 \\
318,15 & 1274,1 & 1303,8 & 1334,6 \\
323,15 & 1271,2 & 1300,9 & 1331,7 \\
328,15 & 1268,3 & 1298,0 & 1328,7 \\
333,15 & 1265,3 & 1295,0 & 1325,7 \\
338,15 & 1262,2 & 1291,9 & 1322,6 \\
343,15 & 1259,1 & 1288,7 & 1319,4 \\
348,15 & 1255,9 & 1285,5 & 1316,2 \\
353,15 & 1252,6 & 1282,2 & 1313,0 \\
\hline Fon
\end{tabular}

Fonte: Autor.

Tabela 3. Massa específica estimada $\left(\mathrm{em} \mathrm{kg} \cdot \mathrm{m}^{3}\right)$ do xarope com $70^{\circ}$ Brix em função da pureza

\begin{tabular}{cccc}
\hline $\begin{array}{c}\text { Temperatura } \\
(\mathrm{K})\end{array}$ & $\begin{array}{c}\text { Pureza } \\
80 \%\end{array}$ & $\begin{array}{c}\text { Pureza } \\
85 \%\end{array}$ & $\begin{array}{c}\text { Pureza } \\
90 \%\end{array}$ \\
\hline 293,15 & 1287,0 & 1317,0 & 1348,0 \\
298,15 & 1284,5 & 1314,4 & 1345,4 \\
303,15 & 1282,0 & 1311,8 & 1342,7 \\
308,15 & 1279,3 & 1309,1 & 1339,9 \\
313,15 & 1276,6 & 1306,4 & 1337,1 \\
318,15 & 1273,8 & 1303,5 & 1334,3 \\
323,15 & 1271,0 & 1300,7 & 1331,4 \\
328,15 & 1268,1 & 1297,7 & 1328,4 \\
333,15 & 1265,1 & 1294,7 & 1325,3 \\
338,15 & 1262,0 & 1291,6 & 1322,2 \\
343,15 & 1258,8 & 1288,4 & 1319,1 \\
348,15 & 1255,6 & 1285,2 & 1315,9 \\
353,15 & 1252,4 & 1282,0 & 1312,6 \\
\hline
\end{tabular}

Fonte: Autor.

Para a obtenção dos valores da energia de ativação para a massa específica $\left(E_{a}\right)$ e da massa específica quando a temperatura tende ao infinito $\left(\rho_{\infty}\right)$ foi empregado o método de linearização de curvas proposto por Freund (2004), na qual a Equação 1 é reescrita na forma de: 


$$
\ln \rho=\ln \rho_{\infty}+\left(\frac{E_{a}}{R}\right) \cdot \frac{1}{T}
$$

Dessa forma, a análise de regressão linear de dados do logaritmo neperiano da massa específica, em função do inverso da temperatura absoluta, fornecem como coeficiente angular da reta obtida a razão $\left(\frac{E_{a}}{R}\right)$ e para o coeficiente linear, o valor do logaritmo neperiano da massa específica quando a temperatura tende ao infinito.

As Equações 3 e 4 determinam, respectivamente, os valores de $\left(\frac{E_{a}}{R}\right)$ e $\ln \rho_{\infty}$, sendo adaptadas do trabalho de Triola (2008).

$$
\frac{E_{a}}{R}=\frac{\left\{n \cdot\left[\sum_{i=1}^{n}\left(\frac{1}{T}\right) \cdot \ln \rho\right]-\left[\sum_{i=1}^{n}\left(\frac{1}{T}\right) \cdot \sum_{i=1}^{n} \ln \rho\right]\right\}}{\left\{n \cdot\left[\sum_{i=1}^{n}\left(\frac{1}{T}\right)^{2}\right]-\left[\sum_{i=1}^{n}\left(\frac{1}{T}\right)\right]^{2}\right\}}
$$

$$
\ln \rho_{\infty}=\frac{\left\{\left(\sum_{i=1}^{n} \ln \rho\right)-\left(\frac{E_{a}}{R}\right) \cdot\left(\sum_{i=1}^{n} \frac{1}{T}\right)\right\}}{n}
$$

A Equação 5 determina o valor do coeficiente de determinação $\left(r^{2}\right)$ para a análise de regressão linear dos dados emparelhados de $\ln \rho$ e $\frac{1}{T}$. Essa equação também foi adaptada do trabalho de Triola (2008).

$$
r^{2}=\frac{\left\{n \cdot \sum_{i=1}^{n}\left\{\left(\frac{1}{T}\right) \cdot(\ln \rho)\right\}-\left\{\left[\sum_{i=1}^{n}\left(\frac{1}{T}\right)\right] \cdot\left[\sum_{i=1}^{n} \ln \rho\right]\right\}\right\}}{\left\{\left\{\left[n \cdot \sum_{i=1}^{n}\left(\frac{1}{T}\right)^{2}\right]-\left[\sum_{i=1}^{n}\left(\frac{1}{T}\right)\right]^{2}\right\}^{1 / 2} \cdot\left\{\left[n \cdot \sum_{i=1}^{n}(\ln \rho)^{2}\right]-\left[\sum_{i=1}^{n} \ln \rho\right]^{2}\right\}^{1 / 2}\right\}}
$$

Com base nos dados indicados nas Tabelas 1,2 e 3, os valores da razão $\left(\frac{E_{a}}{R}\right)$ e $\ln \rho_{\infty}$ foram obtidos a partir da análise de regressão linear dos valores de $\ln \rho$ em função de $\frac{1}{T}$, conforme indicados pelas Equações 3 e 4 .

Nas Equações 3, 4 e 5, $n$ corresponde ao número de dados emparelhados de $\ln \rho$ em função de $\frac{1}{T}$, que de acordo com as Tabelas 1,2 e 3 , equivale a 13.

No cálculo para a determinação dos valores da energia de ativação para a massa específica $\left(E_{a}\right)$, considerou-se que o valor da constante universal dos gases ideais $(R)$ equivale a $8,314 \quad \mathrm{~J} \cdot \mathrm{mol}^{-1} \cdot \mathrm{K}^{-1} \quad(\mathrm{NETZ}$ e ORTEGA, 2008).

\section{RESULTADOS E DISCUSSÃO}

Nas Tabelas 1, 2 e 3 observa-se que à medida em que se aumenta a temperatura, diminui-se a massa específica. Esse comportamento é esperado considerando os comentários realizados por Brown e Holme, (2009).

A Tabela 4 indica os valores encontrados para a razão $\left(\frac{E_{a}}{R}\right), \ln \rho_{\infty}$ e o coeficiente de determinação $\left(r^{2}\right)$. 
O coeficiente de correlação linear $\left(r^{2}\right)$ mede a interdependência linear entre as variáveis e avalia a qualidade do ajuste, ou seja, quanto mais próximo esse coeficiente for da unidade, melhor o ajuste da reta em relação aos pontos da dispersão (PINHEIROS et al., 2009).

Tabela 4. Resultado das análises de regressão linear

\begin{tabular}{|c|c|c|c|}
\hline $\begin{array}{c}\text { Concentração } \\
\text { (Pureza) }\end{array}$ & $\frac{E_{a}}{R}(\mathrm{~K})$ & $\ln \rho_{\infty}$ & $r^{2}$ \\
\hline $\begin{array}{c}60^{\circ} \text { Brix } \\
(80 \%)\end{array}$ & 46,9152 & 7,0018 & 0,9905 \\
\hline $\begin{array}{c}60^{\circ} \mathrm{Brix} \\
(85 \%)\end{array}$ & 46,9250 & 7,0016 & 0,9905 \\
\hline $\begin{array}{c}60^{\circ} \text { Brix } \\
(90 \%)\end{array}$ & 46,9620 & 7,0013 & 0,9906 \\
\hline $\begin{array}{c}65^{\circ} \text { Brix } \\
(80 \%)\end{array}$ & 46,4170 & 7,0266 & 0,9915 \\
\hline $\begin{array}{c}65^{\circ} \text { Brix } \\
(85 \%)\end{array}$ & 46,4460 & 7,0262 & 0,9914 \\
\hline $\begin{array}{c}65^{\circ} \text { Brix } \\
(90 \%)\end{array}$ & 46,4040 & 7,0261 & 0,9915 \\
\hline $\begin{array}{l}70^{\circ} \text { Brix } \\
(80 \%)\end{array}$ & 45,7919 & 7,0520 & 0,9923 \\
\hline $\begin{array}{c}70^{\circ} \text { Brix } \\
(85 \%)\end{array}$ & 45,8090 & 7,0516 & 0,9925 \\
\hline $\begin{array}{c}70^{\circ} \text { Brix } \\
(90 \%)\end{array}$ & 45,8190 & 7,0513 & 0,9923 \\
\hline
\end{tabular}

Fonte: Autor.

Carvalho (2005) comentam que a correlação linear é classificada como muito forte quando os valores do coeficiente de determinação são maiores ou iguais a 0,90 e menores que 1,0 .

Dessa forma, pode-se observar na Tabela 4 que os coeficientes de determinação estão próximos da unidade e que a correlação linear para cada concentração estudada pode ser classificada como muito forte.

A Tabela 5 indica os valores encontrados da energia de ativação para a massa específica $\left(E_{a}\right)$ e da massa específica quando a temperatura tende ao infinito $\left(\rho_{\infty}\right)$.

Tabela 5. Valores da energia de ativação para a massa específica e da massa específica quando a temperatura tende ao infinito

\begin{tabular}{ccc}
\hline $\begin{array}{c}\text { Concentração } \\
(\text { Pureza })\end{array}$ & $E_{a}\left(\mathrm{~J} \cdot \mathrm{mol}^{-1} \cdot \mathrm{K}^{-1}\right.$ & $\rho_{\infty}\left(\mathrm{kg} \cdot \mathrm{m}^{-3}\right)$ \\
\hline $\begin{array}{c}60^{\circ} \mathrm{Brix} \\
(80 \%)\end{array}$ & 390,0530 & 1098,6089 \\
$\begin{array}{c}60^{\circ} \mathrm{Brix} \\
(85 \%)\end{array}$ & 390,1345 & 1098,3892 \\
$60^{\circ} \mathrm{Brix}$ & 390,4421 & 1098,0597 \\
$(90 \%)$ & & \\
$65^{\circ} \mathrm{Brix}$ & 385,9109 & 1126,1950 \\
$(80 \%)$ & & \\
$65^{\circ} \mathrm{Brix}$ & 386,1520 & 1125,7446 \\
$(85 \%)$ & & \\
$65^{\circ} \mathrm{Brix}$ & 385,8029 & 1125,6321 \\
$(90 \%)$ & & 1155,1668 \\
$70^{\circ} \mathrm{Brix}$ & 380,7139 & 1154,7048 \\
$(80 \%)$ & & 1154,3584 \\
$70^{\circ} \mathrm{Brix}$ & 380,8560 & \\
$(85 \%)$ & & \\
$70^{\circ} \mathrm{Brix}$ & 380,9392 & \\
$(90 \%)$ & & \\
\hline
\end{tabular}

Fonte: Autor.

Observa-se na Tabela 5, que aumentando a concentração, para uma mesma pureza, a energia de ativação para a massa específica diminui.

Considerando que a energia de ativação para a massa específica $\left(E_{a}\right)$ indica a sensibilidade da massa específica devido à variação da temperatura, os resultados sugerem que o xarope com concentração de $60^{\circ}$ Brix e pureza de $90 \%$ apresenta uma maior sensibilidade da massa específica em virtude da variação da temperatura, em comparação aos demais xaropes estudados.

A ausência na literatura de valores experimentais da energia de ativação para a 
massa específica $\left(E_{a}\right)$ de xaropes destinados à produção de açúcar e bioetanol dificultou a comparação com os valores encontrados nesse trabalho.

\section{CONCLUSÃO}

O objetivo desse trabalho foi avaliar a influência da temperatura e da concentração na massa específica de xaropes produzidos a partir da evaporação do caldo.

Os valores encontrados para a energia de ativação para a massa específica variaram de $380,7139\left(70^{\circ}\right.$ Brix com $80 \%$ de pureza) a $390,4421 \mathrm{~J} \mathrm{~mol}^{-1} \quad\left(60^{\circ}\right.$ Brix com $90 \%$ de pureza), em um intervalo de temperatura entre 293,15 a 353,15 K.

Os resultados sugerem que com o aumento da concentração, para uma mesma pureza, a energia de ativação para a massa específica diminui. E à medida em que a temperatura aumenta, diminui a massa específica.

Com base nos valores encontrados da energia de ativação para a massa específica, o xarope com concentração de $60^{\circ}$ Brix e pureza de $90 \%$ apresenta uma maior sensibilidade da massa específica em virtude da variação da temperatura, em comparação aos demais xaropes estudados.

$\mathrm{Na}$ literatura, não foram encontrados valores experimentais da energia de ativação para a massa específica de xaropes destinados à produção de açúcar e bioetanol, o que dificultou a comparação dos resultados.

\section{REFERÊNCIAS}

BROWN, L.S.; HOLME, T.A. Química geral aplicada à Engenharia. Cengage Learning, São Paulo, 2009. 653p.

CANCIAM, C.A. Efeito da temperatura na massa específica de ésteres metílicos de ácidos graxos. Revista da Universidade Vale do Rio Verde, v. 12, n. 1, p.17-27, 2014.

CARVALHO, M.J.S. Quimiometrica e espectroscopia no infravermelho próximo (NIR) e médio (MIR) aplicadas na análise de óleos lubrificantes. Dissertação de Mestrado do Programa de Mestrado em Química do Instituto Militar de Engenharia, 2005. 121p.

CHEN, D.; ZHEN, Y.; ZHU, X. Determination of effective moisture diffusivity and drying kinectics for poplar sawdust by thermogravimetric analysis under isothermal condition. Biosource Technology, n. 107, p. 451-455, 2012.

FREUND, J. E. Estatística aplicada Economia, Administração e Contabilidade. Artmed, Porto Alegre, 2004. 545p.

GIAP, S. G. E. The hidden property of Arrhenius-type relationship: viscosity as a function of temperature. Journal of Physical Science, v. 2, n. 1, p. 29-39, 2010.

JÚNIOR, A.C.G.F.; RAMOS, V.H.S.; JESUS, E.; PACÍFICO, J.A. Otimização de produção do caldo clarificado em uma usina de produção de etanol. Scientia Plena, v. 9, n. 3, p. 1-10, 2013.

MAGERRAMOV, M.A.; ABDULAGATOV, A.I.; AZIZOV, N.D.; ABDULAGATOV, I.M. Pressure- and temperature-dependent density change of juices during concentration. 
Food Bioprocess Technology, n.1, p.254-269, 2008.

MATOS, M.A.A. Propriedades termofísicas de alguns gases, sólidos e da água. Editora da Universidade de Aveiro, Aveiro, 1998. 30p.

MEZAROBA, S.; MENEGUETTI, C.C.; GROFF, A.M. Processos de produção do açúcar de cana e os possíveis reaproveitamentos dos subprodutos e resíduos resultantes do sistema. In: IV Encontro de Engenharia de Produção Agroindustrial, 2010, Campo Mourão, Anais Eletrônicos.

NETZ, P.A.; ORTEGA, G.G. Fundamentos de físico-química: uma abordagem conceitual para as ciências farmacêuticas. Artmed, Porto Alegre, 2008. 299p.

PINHEIROS, J.I.D.; CUNHA, S.B.;

CARVAJAL, S.R.; GOMES, G.C. Estatística básica: a arte de trabalhar com dados.

Elsevier, Rio de Janeiro, 2009. 295p.

THE SUGAR ENGINEERS. Disponível em <http:sugartech.co.za/density/index.php>. Acessado em 10 de julho de 2015.

TRIOLA, M.F. Introdução à Estatística. LTC, Rio de Janeiro, 2008. 720p.

TSEN, J.H.; KING, V.A.E. Density of banana puree as a function of soluble solids concentration and temperature. Journal of Food Engineering, v. 55, p.305-308, 2002. 